\title{
Vehicle routing under time-dependent travel times: the impact of congestion avoidance
}

\author{
A.L. Kok*, E.W. Hans, J.M.J. Schutten \\ Operational Methods for Production and Logistics, University of Twente, \\ P.O. Box 217, 7500AE, Enschede, Netherlands
}

\begin{abstract}
Daily traffic congestions form major problems for businesses such as logistical service providers and distribution firms. They cause late arrivals at customers and additional hiring costs for the truck drivers. The additional costs of traffic congestions can be reduced by taking into account and avoid well-predictable traffic congestions within off-line vehicle route plans. In the literature, various strategies are proposed to avoid traffic congestions, such as selecting alternative routes, changing the customer visit sequences, and changing the vehicle-customer assignments. We investigate the impact of these and other congestion avoidance strategies in off-line vehicle route plans on the performance of these plans in reality. For this purpose, we develop a set of VRP instances on real road networks, and a speed model that inhabits the main characteristics of peak hour congestion. The instances are solved for different levels of congestion avoidance using a modified Dijkstra algorithm and a restricted dynamic programming heuristic. Computational experiments show that $99 \%$ of late arrivals at customers can be eliminated if traffic congestions are accounted for off-line. On top of that, almost $70 \%$ of the extra duty times caused by the traffic congestions can be eliminated by clever avoidance strategies.

Keywords: Congestion avoidance; Scenarios; Time-dependent VRP; Time-dependent SPP; Speed model;
\end{abstract}

\footnotetext{
*Corresponding author. Tel.: +31 53489 4164; fax: +31 534892159

E-mail adresses: a.l.kok@utwente.nl, e.w.hans@utwente.nl,j.m.j.schutten@utwente.nl
} 


\section{Introduction}

Due to a growing amount of traffic and a limited capacity of the road network, traffic congestions have become a daily phenomenon. Since traffic congestions cause heavy delays, they are very costly for heavy road users such as logistical service providers and distribution firms. In particular, such delays cause large costs for hiring the truck drivers and the use of extra vehicles, and if they are not accounted for in the route plans they may cause late arrivals at customers or even violations of driving hours regulations. Therefore, accounting for and avoiding traffic congestions has a large potential for cost savings.

Traffic congestion may have several causes. Some are well-predictable, such as the large amount of commuter traffic during the daily peak hours, and others are less predictable, such as the weather or road accidents. Since the peak hour congestions are well-predictable and they constitute a large part (70 to 87\%) of all traffic congestion delays (Skabardonis et al.; 2003), we focus on avoiding peak hour traffic congestions.

Traffic congestions depend on both location and time of the day. Therefore, congestion avoidance is all about not being at the wrong place at the wrong time. There are several strategies to achieve this. For example, changing the visit sequence of a vehicle may avoid large traffic congestions. Another example is to remove a customer from one vehicle route and insert it in another. These congestion avoidance strategies can be optimized by solving a VRP with time-dependent travel times (TDVRP). Although there is a vast literature on the VRP with time-independent travel times (for an extensive overview, see Toth and Vigo (2002)), the literature on the TDVRP is scarce. The only papers we are aware of are: Malandraki and Daskin (1992), Ichoua et al. (2003), Fleischmann et al. (2004), Haghani and Jung (2005), Donati et al. (2008), Van Woensel et al. (2008), and Hashimoto et al. (2008).

Another strategy to avoid traffic congestions is to select an alternative route between two customers at problematic hours. This strategy implies that the route between two customers depends on the chosen departure time, which can be optimized by solving a shortest path problem with timedependent travel times (TDSPP). Orda and Rom (1990) show that solving a time-dependent shortest path problem for a given departure time can be done using a modified Dijkstra (1959) search.

We investigate the impact of different congestion avoidance strategies on the performance of off-line vehicle route plans in a realistic setting. We propose four different scenarios in which congestion avoidance strategies are 
applied to an increasing extent.

To test the impact of the different scenarios in a realistic setting, we develop a number of VRP instances on real road networks and a speed model representing peak hour traffic congestions. This speed model accounts for the main characteristics of peak hour traffic congestion: large delays in urban areas; large delays on road lanes toward urban areas during the morning peak, and in the opposite direction during the evening peak; large delays on roads with a high speed limit (highways). After the route plans are developed in each scenario they are evaluated against the speeds in the road network obtained from the speed model. This methodology evaluates the impact of the different levels of congestion avoidance in reality.

The contributions of this paper are the following. First, to the best of our knowledge, this is the first paper which inhabits the full complexity of solving vehicle routing problems and shortest path problems on real road networks with traffic congestions. Second, this paper quantifies the performance improvement for both duty times and travel distances by accounting for and avoiding traffic congestions. Due to the simplicity of many of the vehicle routing models proposed in the literature (in general, client networks are considered in which travel distances are set to euclidean distances and travel times are proportional to these travel distances), this quantification is impossible within these models. Third, this paper quantifies the number of late arrivals and the late times at customers as a consequence of a lack of accounting for and avoiding traffic congestions. Since this is done for different congestion avoidance strategies, we get a clear indication of which congestion avoidance strategies are necessary or beneficial in gaining high quality vehicle route plans. Fourth, this paper proposes a speed model on large road networks which inhabits the main characteristics of peak hour traffic congestions. Therefore, it forms a valuable platform for quantifying performance improvements by new vehicle routing models and solution methods, as well as shortest path algorithms.

This paper is organized as follows. In Section 2, we propose a speed model representing the main characteristics of peak hour traffic congestions. In Section 3, we formalize several congestion avoidance strategies in four scenarios for solving off-line vehicle routing problems and in Section 4, we propose a general solution approach to solve the problem instances in these four scenarios. In Section 5, we present the impact of the different scenarios on the real-life performance and in Section 6, we give some concluding remarks. 


\section{Speed model}

To investigate the impact of the different congestion avoidance strategies in a realistic setting, we propose a speed model for real road networks that reflects the main characteristics of peak hour traffic congestions. The speed model is designed for the road network data used in this paper, but the methodology can be applied to any other road network data.

Our road network data is a selection of the TIGER/Line files (Tiger/line; 2002), which consist of road network data of each of the 50 US states. We select the states Rhode Island, Connecticut, Maryland, Massachusetts, and New Jersey, because they have a high degree of urbanization overall, resulting in many traffic congestions. On top of that, the sizes of these states are comparable to some smaller countries in Europe such as the Netherlands and Belgium, which have to face large congestion problems since they are densely populated. Furthermore, we select Kentucky for comparison reasons: this state has next to a relatively small urban area also large rural areas.

The TIGER/Line data contain geoinformation on nodes in the road network (a node may represent an intersection of different roads or a change in average speed on the same road), and distance and road category information on directed arcs connecting these nodes. There are four different road categories with their corresponding average speeds. These speeds are time-independent.

We propose a time-dependent speed model which incorporates peak hour traffic congestions. This speed model defines for each arc a speed at which the arc is traversed during the peak hours and a speed at which the arc is traversed outside the peak hours. The speed outside the peak hours is set to the speed provided by the TIGER/Line files. The speed drop downs during the peak hours are based on the main characteristics of peak hour traffic congestions.

The peak hour congestions are mainly caused by a large amount of commuter traffic. Since commuter traffic needs to be at the same time (at the start of the working day) at the same place (large cities), the most common roads get silted up during the peak hours. We observe three main characteristics of peak hour traffic congestion:

1. Degree of urbanization. Within urban areas, there is much more traffic congestion than in rural areas. Therefore, there is a positive relation between the degree of urbanization and the speed drop downs during 
the peak hours.

2. Direction of commuter traffic. During the morning peak, commuter traffic is traveling toward working areas. Therefore, during the morning peak much more traffic congestion appears on road lanes directed to urban areas than on road lanes in the opposite direction (and during the evening peak vice versa).

3. Speed limit. In general, roads with a high speed limit (highways) are more heavily used than roads with a lower speed limit (rural roads). Because these roads lie on many (free-flow) shortest paths, there is a positive relation between a road's speed limit and the amount of traffic congestion during the peak hours.

We propose the following approach to quantify the speed drop downs during the peak hours on each arc in the road network, based on the three observations described above. First we determine the degree of urbanization of the source- and destination-node of the arc under consideration. We determine this, by counting the number of network nodes in the proximity area of each node. We refer to such nodes as proximity nodes. In Section 2.1, we explain in detail how this proximity area is defined and how we use it to determine the degree of urbanization of each node. We set the degree of urbanization of each arc to the maximum of the degrees of its source- and destination-node. Next, we determine the direction of the arc, i.e., toward or from an urban area. If the destination-node has a higher number of proximity nodes than the source-node, then the arc is directed toward an urban area. Finally, the speed limit on the arc is given by the road category of the arc under consideration.

Using the calculated degree of urbanization, the arc direction, and the road category, we calculate the speed drop downs as follows. Table 1 presents the maximum (relative) speed drop downs during the morning peak (for the evening peak the two rows must be swapped). These maximum speed drop downs depend both on the arc direction and on the road category. We multiply the corresponding speed drop down with a fraction, depending on the degree of urbanization. Table 2 presents these fractions. Degree 1 represents the highest degree of urbanization.

Using this approach, the speed model inhabits all three main characteristics of peak hour traffic congestion. Table 1 presents the dependency of the speed drop downs on the road direction and the speed limit, and Table 


\begin{tabular}{l||c|c|c|c} 
& road cat. 1 & road cat. 2 & road cat. 3 & road cat. 4 \\
\hline $\begin{array}{l}\text { Arcs toward } \\
\text { urban areas }\end{array}$ & 0.9 & 0.65 & 0.4 & 0.15 \\
\hline $\begin{array}{l}\text { Arcs from } \\
\text { urban areas }\end{array}$ & 0.3 & 0.25 & 0.2 & 0.15 \\
\hline
\end{tabular}

Table 1: Maximum speed drop downs during the morning peak

\begin{tabular}{c|c} 
Degree of urbanization & Fraction of speed drop down \\
\hline 1 & 1 \\
2 & $2 / 3$ \\
3 & $1 / 3$ \\
4 & 0
\end{tabular}

Table 2: Degree of urbanization and corresponding speed drop down fraction

2 shows that arcs located in high degrees of urbanization encounter larger speed drop downs than arcs in low degrees of urbanization.

\subsection{Determining the degree of urbanization of a node}

We propose the following methodology for determining the degree of urbanization of each node in the road network. We define the proximity area of a node to a circle with a radius of $10 \mathrm{~km}$, such that urban areas are identified if a node is in a $10 \mathrm{~km}$ range of this urban area. To get an indication of the number of proximity nodes for a node that lies in the center of a large city and, therefore, has the highest degree of urbanization, we determine for each state the maximum number of proximity nodes over all nodes that lie in the largest city of that state.

Table 3 shows that nodes which have the largest degree of urbanization contain 15 thousand or more proximity nodes. Therefore, we set the degree of urbanization of a node to 1 if it contains at least 15 thousand proximity nodes. The numbers of proximity nodes corresponding to the other degrees of urbanization are evenly spread between 0 and 15 thousand. Table 4 presents the resulting correspondence between the number of proximity nodes and the degree of urbanization.

For each arc, this speed model defines the speed drop down during the morning peak and evening peak. The road networks together with the speed 


\begin{tabular}{l||c} 
State & $\begin{array}{c}\text { Max \# proximity } \\
\text { nodes }(\times 1000)\end{array}$ \\
\hline Connecticut & 15 \\
Kentucky & 19 \\
Rhode Island & 24 \\
Maryland & 28 \\
New Jersey & 32 \\
Massachusetts & 38
\end{tabular}

Table 3: Maximum \# proximity nodes in the largest city

\begin{tabular}{c|c}
$\begin{array}{c}\text { proximity nodes } \\
(\times 1000)\end{array}$ & degree of urbanization \\
\hline $15+$ & 1 \\
$10-15$ & 2 \\
$5-10$ & 3 \\
$0-5$ & 4
\end{tabular}

Table 4: Number of proximity nodes and degree of urbanization

model form a suitable platform to generate realistic VRP instances. In Section 5, we use these instances to test the real-life performance of different congestion avoidance strategies in off-line vehicle route plans. In Section 3, we formalize such congestion avoidance strategies in four different scenarios.

\section{Scenarios}

We propose four different scenarios in which congestion avoidance strategies are applied to an increasing extent. The first two scenarios are closest to the VRP literature, in which the travel times are modeled as time-independent. The only difference between these scenarios is that Scenario 2 accounts to some extent for traffic congestions, while Scenario 1 completely ignores the traffic congestions. In the other two scenarios time-dependent travel times are accounted for and, therefore, traffic congestions can be avoided. This is done to a greater extent in Scenario 4 than in Scenario 3. Each scenario consist of two phases. In phase one the (time-dependent) shortest paths are determined between the customers. In phase two a (time-dependent) VRP is solved, based on the travel times resulting from phase one. We now describe 
the four scenarios in more detail.

Scenario 1 is the base scenario in which traffic congestions are completely ignored. In phase one, all free-flow shortest paths between the customers are determined. For this purpose, the speeds at the arcs in the road network are set to their maximum speed of the day (the speed outside the peak hours). The travel times along the paths are calculated using these maximum speeds. Next, a VRP is solved based on the resulting free-flow travel times between the customers. This scenario does not account for traffic congestions to any extent, and therefore no congestion avoidance strategy is applied.

In Scenario 2, traffic congestions are accounted for by solving a VRP based on average travel times over the day. As in Scenario 1, the free-flow shortest paths are determined in the first phase by setting all arcs to their maximum speed. These paths are fixed and they will be used if they are selected in phase two. Next, the average travel times along these paths are determined by evaluating the exact travel times for a large number of different departure times (we set the inter-departure time to 15 minutes). For each departure time, the travel times are calculated using the time-dependent arc speeds resulting from the speed model. The averages over these time-dependent travel times are used as input for a VRP in phase two. In this scenario, traffic congestions are accounted for by averaging the delays along a route over the entire day. Traffic congestions could be avoided to a small extent if routes with smaller average travel times are selected due to smaller delays during the peak hours.

In Scenario 3, traffic congestions are avoided by solving a TDVRP. The time-dependent travel times which form the input for the TDVRP are determined in a similar way as in Scenario 2. The only difference is that the large number of time-dependent travel times (with inter-departure times of 15 minutes) is not averaged over the day. Interpolation is used each time the TDVRP solver requires the travel time between two customers for a given departure time. Note that in this scenario the routes between the customers are still fixed at the free-flow shortest paths. This scenario accounts for traffic congestions to a high extent and allows congestion avoidance by selecting alternative customer visit sequences and alternative vehicle-customer assignments.

Scenario 4 adopts the highest level of congestion avoidance. In this scenario, time-dependent shortest paths are determined for a large number of different departure times in phase 1 (the inter-departure times are again set at 15 minutes). Next, the time-dependent travel times are determined for 
all these different departure times along the corresponding time-dependent shortest paths and used as an input for solving a TDVRP. Again, interpolation is used to estimate the travel time for a given departure time when the TDVRP is being solved. Finally, since the resulting planned departure times generally do not coincide with a departure time for which the time-dependent shortest path has already been determined in phase 1 , we determine the shortest paths for the planned departure times as a last step in this scenario. This scenario accounts for traffic congestions to the same extent as Scenario 3, but adopts a higher level of congestion avoidance: at problematic hours alternative routes between customers are chosen. Table 5 gives an overview of the four scenarios.

\begin{tabular}{l||l|l|l|l} 
& $\begin{array}{l}\text { Shortest } \\
\text { paths }\end{array}$ & $\begin{array}{l}\text { Travel times } \\
\text { input for VRP }\end{array}$ & $\begin{array}{l}\text { Accounting for } \\
\text { congestions }\end{array}$ & $\begin{array}{l}\text { Avoiding } \\
\text { congestions }\end{array}$ \\
\hline Scen. 1 & free-flow & free-flow & no & no \\
Scen. 2 & free-flow & average & a small extent & no \\
Scen. 3 & free-flow & time-dep. & yes & yes \\
Scen. 4 & time-dep. & time-dep. & yes & yes
\end{tabular}

Table 5: Scenario overview

After the VRP instances are solved in each scenario, the performances of the resulting vehicle route plans are evaluated according to the speeds resulting from the speed model in the road network. Note that in each scenario travel time estimations are used to solve a (TD)VRP. The reason for using travel time estimations in Scenario 3 and 4 is that calculating the exact travel time based on the chosen route and the speed model each time a travel time is needed would be too time-consuming. On top of that, these travel time calculations depend on the number of arcs along the route under consideration and, therefore, on the size of the road network. In practice, however, the TDVRP often needs to be solved within limited computation times. Therefore, the proposed travel time estimations, which do not depend on the size of the road network, are suitable for practice.

The required computation times to solve the shortest path problems also depend on the size of the road networks. However, in practice shortest paths are re-used many times: only the addition of new customers or road network changes require shortest path (re-)calculations. Therefore, these shortest paths can often be determined in a pre-processing phase in which computation times play a minor role. 


\section{Solution methods}

In each scenario, we need to solve a (TD)SPP and a (TD)VRP. To make a fair comparison of the different scenarios, we solve the problems with solution methods that do not need to be tailored for each specific problem. This means that the shortest path algorithm can solve both SPPs and TDSPPs, and the VRP algorithm can solve both VRPs and TDVRPs. We also require that the computation times of the VRP solution methods are (approximately) the same for each scenario, such that this does not affect the applicability of the different scenarios in practice. For the shortest path algorithms we are less restrictive, since shortest path calculations are generally done as a pre-processing in practice.

We solve the (TD)SPPs with a modified Dijkstra (1959) algorithm. The only adaptation we make is that we initiate the searches with a given departure time from the source node, and we keep track of the departure times at each reached node. This is necessary to determine the time-dependent travel times when the labels of the nodes need to be updated. This approach allows us to solve the shortest path problems in Scenarios 1 to 3 in (approximately) the same computation times. Only in Scenario 4 the computation time increases, since we have to rerun the algorithm for each possible departure time. However, this is all done in phase 1, a pre-processing phase in which computation times play a minor role in practice.

We solve the (TD)VRPs with a restricted dynamic programming heuristic, introduced by Gromicho et al. (2008). We select this solution method, since it can solve VRPs and TDVRPs with similar computation times. Moreover, no tailoring is necessary for the problems at hand. This is not only very valuable for practical use, but it also provides a fair comparison of the quality of the different scenarios. Finally, this method has the great advantage that it can solve small problem instances to optimality, and only when computation times become restrictive it acts as a heuristic.

\section{Computational experiments}

We test the impact of the four scenarios of congestion avoidance on a large number of VRP instances. These VRP instances are developed on the road networks of the six selected states and the speeds resulting from the speed model. The customer locations are uniformly randomly selected from the 
nodes in the road network. Clustering of the customer locations is a natural result, since the road network is denser in urban areas. Furthermore, the first selected node is considered to be the depot.

We develop 15, 50, and 100 customer problem instances. The 15 customer problem instances are small enough to be solved to optimality in practical computation times. The 100 customer problem instances are approximately the largest instances for which we can still solve a sufficiently large number of problem instances within practical computation times.

We add hard time windows to 50 percent of the customers indicating the period in which service must start. We set the time window of the depot to $[0,14]$, indicating a working day of 14 hours. The width of the time windows at the selected customers are randomly drawn from $\{2,3,4,5,6\}$ quarters of an hour. The customer service times are randomly drawn from $\{1,2\}$ quarters of an hour and the demands are randomly drawn from $\{1,2, \ldots, 10\}$. Finally, we set the vehicle capacities as follows. If the vehicle capacities are set too low, then the length of vehicle routes are only restricted by these capacities. If they are set too high, then the length of the routes are only restricted by the time windows. Initial experiments showed that a capacity of 55 results in a good trade off and is therefore used in our experiments. We generate 20 problem instances for each combination of state and number of customers resulting in $360 \mathrm{VRP}$ instances in total.

The primary objective is set to minimize the total number of vehicle routes, and the secondary objective to minimize the total duty time of the truck drivers. Duty times are the sum of travel, service, and waiting times. We choose to minimize duty times as a secondary objective instead of travel times or travel distances, since small duty times are generally more important in practice, as duty times define the hiring costs of the truck drivers and the periods in which the trucks are not available for other services.

We choose to dispatch the trucks at time zero. Although postponing the departure times at the depot may significantly reduce duty times (Kok et al.; 2008), it is beyond the scope of this paper to also optimize the departure times of the vehicles. Therefore, extending this study with the impact of departure optimization on the performance of vehicle route plans is one of our recommendations for future research.

We implemented the data-structures and solution algorithms in Delphi 7 on a Pentium 4, 3.40GHz CPU and $1.00 \mathrm{~GB}$ of RAM. Table 6 presents the main average results of the four different scenarios aggregated over all problem instances (we aggregate the average results of the 15-, and 50-customer 
instances to 100-customer instances), except for the problem instances generated on Kentucky. We only present aggregated results, because the average results of the 15-, 50-, and 100-customer problem instances are similar. The relative differences of the performance measures of the last three scenarios with respect to the first scenario are presented in Table 7 . All performance measures are derived by evaluating the developed route plans in each scenario against the speeds resulting from the speed model. Next to the two objectives 'minimizing number of vehicle routes' and 'minimizing duty times', we also report on the following performance measures: total travel distance, total number of late arrivals at customers, total number of late return times at the depot, maximum late time over all customers, and total late time over all customers. Although we do not optimize on travel distances, we also report the impacts of the scenarios on this performance measure. In traditional VRP models in which customer networks are considered instead of road networks it is not possible to distinguish between duty times and travel distances. However, for practice both measures are relevant, since they cause different transport costs. The last four performance measures indicate the reliability of the route plans. Note that all performance measures present aggregated averages over all problem instances, except for 'Maximum late time' which presents the maximum over all problem instances.

\begin{tabular}{l||c|c|c|c} 
Performance measure & Scen. 1 & Scen. 2 & Scen. 3 & Scen. 4 \\
\hline \# vehicle routes & 12.18 & 12.65 & 12.31 & 12.23 \\
Total duty time (hours) & 124.8 & 126.9 & 123.2 & 122.0 \\
Total travel distance & 49.37 & 48.74 & 50.32 & 50.00 \\
\# late arrivals & 12.36 & 3.068 & 0.070 & 0.062 \\
\# late return times & 0.364 & 0.117 & 0.000 & 0.000 \\
Maximum late time (hours) & 3.215 & 1.396 & 0.554 & 0.034 \\
Total late time (hours) & 7.310 & 0.962 & 0.008 & 0.001
\end{tabular}

Table 6: Main results, aggregated over all problem instances

The number of vehicle routes is larger in Scenarios 2, 3 and 4 than in Scenario 1, since the travel time estimations in Scenario 1 are lower bounds to the travel times estimations in the other three scenarios. The reason is that the VRPs solved in Scenario 1 are based on free-flow shortest paths with the corresponding free flow travel times. However, the results indicate that avoiding traffic congestion to an increasing level reduces the number of vehicles again and approaches the number of vehicles required in Scenario 1. 


\begin{tabular}{l||r|r|r} 
Performance measure & Scen. 2 & Scen. 3 & Scen. 4 \\
\hline \# vehicle routes & $3.90 \%$ & $1.12 \%$ & $0.40 \%$ \\
Total duty time (hours) & $1.73 \%$ & $-1.24 \%$ & $-2.24 \%$ \\
Total travel distance & $-1.28 \%$ & $1.94 \%$ & $1.29 \%$ \\
\# late arrivals & $-75.17 \%$ & $-99.43 \%$ & $-99.50 \%$ \\
\# late return times & $-67.94 \%$ & $-100.00 \%$ & $-100.00 \%$ \\
Maximum late time (hours) & $-56.57 \%$ & $-82.77 \%$ & $-98.94 \%$ \\
Total late time (hours) & $-86.83 \%$ & $-99.89 \%$ & $-99.98 \%$
\end{tabular}

Table 7: Scenarios 2, 3 and 4 relative to Scenario 1

The duty times show a similar pattern, which can be partially explained by the number of vehicle routes. Furthermore, the additional congestion information in Scenarios 2, 3 and 4 with respect to Scenario 1 reduces the duty times. This even results in an overall decrease of duty times in Scenarios 3 and 4, despite the larger number of vehicle routes. Note that if there were no traffic congestions, Scenario 1 would give the best solutions. Therefore, if we subtract the estimated duty times in Scenario 1 (which are based on freeflow travel times) from the real duty times in each scenario, then we obtain estimations of the additional duty times caused by the traffic congestions. Table 8 presents the aggregated average amount of additional duty time for each scenario with respect to the estimated duty times in Scenario 1. The results show that Scenario 2 has larger additional duty times than Scenario 1, but Scenarios 3 and 4 reduce the additional duty times by $45 \%$ and $67 \%$, respectively.

\begin{tabular}{l||c|c|c|c} 
& Scen. 1 & Scen. 2 & Scen. 3 & Scen. 4 \\
\hline Additional duty time & 5.658 & 7.173 & 3.141 & 1.885
\end{tabular}

Table 8: Average additional duty time caused by traffic congestions

The travel distances show a different pattern, which is a bit harder to explain. Due to the additional congestion information in Scenario 2, routes with a small average delay over the day are more interesting than in Scenario 1. These routes typically contain many roads with a small maximum speed. However, these routes are only selected in the VRP if the smaller maximum speeds are compensated by smaller travel distances, resulting in smaller travel distances in Scenario 2 than in Scenario 1. In Scenarios 3 and 4, routes with many arcs with a high maximum speed can still be interesting, even 
if the distances are also higher, because the additional traffic congestion information on the timing of these delays make that these routes can be selected at convenient departure times. At such departure times, the extra distances are compensated by the high travel speeds, resulting in larger travel distances than in Scenarios 1 and 2.

The reliability of the route plans strongly increase if the level of congestion avoidance increases. All reliability measures show a strong reduction with respect to Scenario 1. This is not surprising, since the scenarios account for traffic congestions to an increasing level. However, the huge improvement of Scenario 2 with respect to Scenario 1 in comparison with the additional improvements of the other two scenarios is less obvious. The explanation for this is that an underestimation of an early travel in a vehicle route in Scenario 1 propagates along all arrival times at successive customers of that vehicle route. In Scenario 2, such an underestimation of an early travel is generally compensated by an overestimation on a later travel. Another surprising result is the additional reliability improvement of Scenario 4 with respect to Scenario 3. This improvement is only caused by an higher level of congestion avoidance, not by accounting for traffic congestions to a higher extent.

Table 9 presents the relative differences of Scenarios 2, 3 and 4 with respect to Scenario 1 for the state Kentucky. As mentioned before, Kentucky contains a very large rural area compared to the other 5 states. Therefore, the main part of the routes of the problem instances generated on this state do not contain delays caused by traffic congestions. Table 9 shows that this has a strong impact on the results. The underestimation of the travel times in Scenario 1 hardly leads to a smaller number of routes than in the other scenarios. The differences in duty times are even negligible. The reliability measures show similar results as at the other states. However, it should be mentioned that in Scenario 1 the average number of late arrivals is 1.9 (of the 50 customers with time windows), the average number of late return times is 0.08 , the maximum late time is 1.06 hours, and the average total late time is 0.61 hours. Therefore, the route plans in Scenario 1 for Kentucky are already much more reliable than the route plans in Scenario 1 for the other states. 


\begin{tabular}{l||r|r|r} 
Performance measure & Scen. 2 & Scen. 3 & Scen. 4 \\
\hline \# vehicle routes & $0.14 \%$ & $0.41 \%$ & $0.27 \%$ \\
Total duty time (hours) & $-0.07 \%$ & $-0.03 \%$ & $-0.07 \%$ \\
Total travel distance & $0.08 \%$ & $0.89 \%$ & $2.70 \%$ \\
\# late arrivals & $-81.75 \%$ & $-100.00 \%$ & $-96.92 \%$ \\
\# late return times & $-50.00 \%$ & $-100.00 \%$ & $-100.00 \%$ \\
Maximum late time (hours) & $-51.04 \%$ & $-100.00 \%$ & $-99.49 \%$ \\
Total late time (hours) & $-89.80 \%$ & $-100.00 \%$ & $-99.96 \%$
\end{tabular}

Table 9: Change of scenarios 2, 3 and 4, relative to Scenario 1 for Kentucky

\section{Conclusions}

We proposed four different scenarios in which traffic congestions are accounted for and avoided to an increasing extent. Next, we proposed a speed model on real road networks, which inhabits the main characteristics of peak hours traffic congestions. This speed model provided us a platform for testing the impact of the different scenarios in reality.

The results indicated that the reliability of route plans strongly increase if traffic congestions are accounted for. However, if VRPs are modeled with time-independent travel times, then this reliability increase is achieved against the cost of increasing cost measures such as number of vehicle routes and total duty time. By adopting higher levels of congestion avoidance such as solving VRPs with time-dependent travel times and solving timedependent shortest path problems, these cost measures can be reduced significantly. Solving a combination of these two problems is particularly effective, resulting in huge reliability improvements, significant duty time reductions (almost two third of the additional duty times caused by traffic congestions can be eliminated), and significantly reducing the number of vehicles needed (almost all extra vehicles needed due to congestion delays can be eliminated).

\section{Acknowledgment}

This work was financially supported by Stichting Transumo through the project ketensynchronisatie. 


\section{References}

Dijkstra, E. W. (1959). A note on two problems in connexion with graphs, Numerische Mathematik pp. 269-271.

Donati, A. V., Montemanni, R., Casagrande, N., Rizzoli, A. E. and Gambardella, L. M. (2008). Time dependent vehicle routing problem with a multi ant colony system, European journal of operational research 185(3): 1174-1191.

Fleischmann, B., Gietz, M. and Gnutzmann, S. (2004). Time-varying travel times in vehicle routing, Transportation science 38(2): 160-173.

Gromicho, J., van Hoorn, J., Kok, A. L. and Schutten, J. M. J. (2008). The flexibility of restricted dynamic programming for the vrp, Beta working paper series $\mathbf{2 6 6}$.

Haghani, A. and Jung, S. (2005). A dynamic vehicle routing problem with time-dependent travel times, Computers $\&$ operations research 32(11): 2959-2986.

Hashimoto, H., Yagiura, M. and Ibaraki, T. (2008). An iterated local search algorithm for the time-dependent vehicle routing problem with time windows, Discrete Optimization 5: 434-456.

Ichoua, S., Gendreau, M. and Potvin, J. (2003). Vehicle dispatching with time-dependent travel times, European journal of operational research 144(2): 379-396.

Kok, A. L., Hans, E. W. and Schutten, J. M. J. (2008). Optimizing departure times in vehicle routes, Beta working paper series 236.

Malandraki, C. and Daskin, M. (1992). Time dependent vehicle routing problems: Formulations, properties and heuristic algorithms, Transportation science 26(3): 185-200.

Orda, A. and Rom, R. (1990). Shortest-path and minimum-delay algorithms in networks with time-dependent edge-length, Journal of the Association for Computing Machinery 37(3): 607-625. 
Skabardonis, A., Varaiya, P. and Petty, K. (2003). Measuring recurrent and nonrecurrent traffic congestion, Transportation Research Record 1856: $118-124$.

Tiger/line (2002). United states road networks. http://www . dis . uniroma1 . it/ challenge9/data/tiger/.

Toth, P. and Vigo, D. (2002). The vehicle routing problem, SIAM Monographs on Discrete Mathematics and Applications, Philadelphia.

Van Woensel, T., Kerbache, L., Peremans, H. and Vandaele, N. (2008). Vehicle routing with dynamic travel times: A queueing approach, European journal of operational research 186(3): 990-1007. 